\title{
On the Validity of the Master Equation for Finite Closed Systems
}

O. Seeberg

Institut für Theoretische Physik der Johann Wolfgang Goethe-Universität, Frankfurt am Main, Germany

Z. Naturforsch. 33a, 627-637 (1978); received March 8, 1978

Two equations for the macroscopic part $W$ of the statistical operator are considered:

1. the master equation $\dot{W}=-\boldsymbol{M} W$,

2. the exact equation $\dot{W}=-\int_{0}^{t} \boldsymbol{K}(t-\tau) W(\tau) \mathrm{d} \tau$.

It follows from the physical equivalence of the solutions together with a stability assumption and the assumption that there is a time $\tau^{*}$ after which also the derivatives of the solutions are equivalent, that $\tau^{*}$ is the life-time of the kernel $\boldsymbol{K}$ and that $\boldsymbol{M}=\int_{0}^{\tau *} \boldsymbol{K}(\tau) \mathrm{d} \tau$. Conversely, the equivalence of the solutions follows from assumptions on the life-time of the kernel $\boldsymbol{K}$ together with a stability assumption and a smoothness assumption on the initial statistical operator $W(0)$.

\section{Introduction}

It is the aim of this paper to investigate the validity of the master equation for finite systems. Thus all limiting processes are to be excluded from the considerations. This investigation is of interest within the framework of the theory of dynamical critical phenomena, where some of the assumptions discussed in this paper may break down.

Our starting point (II) is a definition of "validity". It is well-known that a master equation cannot hold in a mathematical sense, this follows from the reversibility of the underlying microscopic theory (quantum mechanics or classical mechanics). Hence a weaker definition of validity must be introduced which - furthermore - takes into account the recurrence theorem. The validity only can be demanded for finite times of the magnitude of the relaxation time. We introduce the meaning of the physical equivalence of two ensembles by comparing the expectation values of a given set of macroscopic observables. This comparison depends on the measurement devices and their scales, thus a constant $\varepsilon$ measuring the accuracy of the scales will appear in all considerations. The choice $\varepsilon=0$ yields a much more simple definition but this definition is without physical sense. Now we assume quantum mechanics to be true and then we get by well-known projection techniques an exact equation of motion for the macroscopic part of the statistical operator: $\dot{W}=-\int_{0}^{t} \boldsymbol{K}(t-\tau) W(\tau) \mathrm{d} \tau$. This equation is a nonlocal reversible equation while the master equation $\dot{W}=-\boldsymbol{M} W$ is a local irreversible equation with semigroup property. Starting with identical initial ensembles we get an equation for the difference of the two solutions leading to a formal operator identity (III). This identity is the basis of the following considerations. In Part IV we at first list some relations which are used as presuppositions for our proofs. Such presuppositions must be introduced, because we only make use of the form of the exact equation, that means of the existence of the kernel $\boldsymbol{K}$. Thus we give no "derivation" of the master equation from first principles. Instead of that we investigate the conclusions from the physical equivalence of the solutions and conversely discuss some assumptions which lead to that equivalence. It turns out that four groups of assumptions must be introduced. The first group contains assumptions on the finite life-time $\tau^{*}$ of the kernel $\boldsymbol{K}$. If these assumptions are not valid for all operators $A \in \Re, \Re$ being the space of the macroobservables, then we can define sets of operators by these properties. Then the second group contains closure assumptions for these sets. The third group consists of stability assumptions for the basic equations and the last group contains assumptions on the properties of the initial statistical operators, these are smoothness assumptions.

The main result is that a certain combination of these assumptions leads to the physical equivalence of the solutions, while physical equivalence of the solutions together with the physical equivalence of the temporal derivatives of these solutions for times $t \geqq \tau^{*}$, an additional closure property and an additional stability property yield some finite 
life-time properties of the kernel $\boldsymbol{K}$ and

$$
\boldsymbol{M} \cong \int_{0}^{\tau^{*}} \boldsymbol{K}(\tau) \mathrm{d} \tau
$$

Furthermore we get a condition of nonergodicity following from the form of the kernel $\boldsymbol{K}$.

A very similar combination of assumptions together with a temporal smoothing process yield the result that the smooth part of the exact solution obeys a master equation. If in addition a smoothness property for $W(0)$ is valid, then the equivalence of the solutions follows.

From a physical point of view the assumptions of stability must be fulfilled. If this were not the case, the basic equations would be without physical sense. These equations govern the behaviour of ensembles, not of single systems.

The conditions of finite life-time depend on the relations between the Hamiltonian $H$ and the macroscopic observables and on the choice of the energy shell $\mathfrak{\mathfrak { H }}$. The investigation of these relations would be part of a complete theory of macroscopic observables.

\section{Physical Equivalence}

Let us consider a microcanonical energy shell $\mathfrak{h}$ :

$$
\mathfrak{H}=\left\{\varphi_{\nu} \mid H \varphi_{\nu}=\varepsilon_{\nu} \varphi_{\nu}, E-\Delta E \leqq \varepsilon_{\nu}<E\right\} .
$$

$H$ is the Hamiltonian.

$\mathfrak{H}$ can be regarded as a finite dimensional Hilbert space, $\operatorname{dim} \mathfrak{S}=d$. Let be $\mathfrak{Q}$ the space of the linear operators on $\mathfrak{h}, \Omega$ a subspace of macroscopic observables. $\mathfrak{Q}$ becomes a Hilbert space again by introduction of the trace product [1]:

$$
(A ; B)=\operatorname{Sp}\left(A^{+} B\right) .
$$

We assume that $\Re$ is spanned by a basis of projection operators $P_{i}$ onto orthogonal subspaces

$$
\mathfrak{r}_{i} \subset \mathfrak{H}, \quad \operatorname{dim} \mathfrak{r}_{i}=d_{i} .
$$

Then the projection $W=\boldsymbol{G} U$ of the statistical operator $U$ onto $\Re$ always is a positive operator (Appendix A). This latter property is essential for the linearity of the equations of motion for the expectation values $\left\langle A_{i}\right\rangle, A_{i} \in \Re$ [2]. Furthermore we assume that $P_{\mathfrak{g}} \in \Re$.

Let us now consider two different equations of motion. The first equation is a master equation:

$$
\dot{\tilde{W}}=-\boldsymbol{M} \tilde{W}, \quad \tilde{W} \in \Re .
$$

The eigenvalue problem is: $\boldsymbol{M} O_{i}=\lambda_{i} O_{i}$. We assume that

1. The $O_{i}$ form a basis in $\Re$,

2. $\operatorname{Re} \lambda_{i} \geqq 0$,

3. $\operatorname{Re} \lambda_{i}=0 \Rightarrow \lambda_{i}=0, \quad O_{i}=\gamma P_{5}$.

The second equation is the exact equation:

$$
\begin{aligned}
\dot{W}= & -\int_{0}^{t} \boldsymbol{K}(t-\tau) W(\tau) \mathrm{d} \tau, \\
\boldsymbol{K}(t)= & \boldsymbol{G L}(\mathbf{1}-\boldsymbol{G}) \\
& \cdot \exp [i(\mathbf{1}-\boldsymbol{G}) \boldsymbol{L}(\mathbf{1}-\boldsymbol{G}) t](\mathbf{1}-\boldsymbol{G}) \boldsymbol{L} \boldsymbol{G} .
\end{aligned}
$$

This equation is derived with the help of the Zwanzig projection technique $[3,4]$ and the assumption that $\boldsymbol{G} U(0)=U(0)$. The latter assumption requires that $\boldsymbol{G} U(0)>0$. Now, the question arises under which circumstances the solutions of Eq. (4) and Eq. (6) do agree. Of course there cannot be an exact equality. Therefore we must introduce a new definition. The statistical operators $W_{1}, W_{2}$ are called physically equivalent, $W_{1} \sim W_{2}$, if the corresponding ensembles cannot be discriminated by macroscopic measurements. That means:

$$
\left|\operatorname{Sp}\left[\left(W_{1}-W_{2}\right) A_{i}\right]\right| \leqq \eta_{i}
$$

where $\eta_{i}$ is the scale length of the corresponding apparatus $\eta_{i}$. Thus we get the following sufficient condition for physical equivalence:

$$
\begin{aligned}
& \left|\operatorname{Sp}\left[\left(W_{1}-W_{2}\right) A_{i}\right]\right|=\left|\left(W_{1}-W_{2} ; A_{i}\right)\right| \\
& \leqq\left\|W_{1}-W_{2}\right\|\left\|A_{i}\right\| \leqq \eta_{i} .
\end{aligned}
$$

So we are led to the following stronger definition:

$$
\begin{aligned}
& W_{1} \sim W_{2}, \quad \text { if }\left\|W_{1}-W_{2}\right\| \leqq \varepsilon, \\
& \varepsilon=\min \eta_{i} /\left\|A_{i}\right\| .
\end{aligned}
$$

The norm $\|X\|$ is defined by $\|X\|^{2}=\operatorname{Sp}\left(X^{+} X\right)$. We emphasize that "physical equivalence" does not imply an equivalence relation. And a second remark: From $\|W\| \leqq 1$ it follows that

$$
\left\|W_{1}-W_{2}\right\| \leqq \varepsilon\left\|W_{1}\right\| \Rightarrow W_{1} \sim W_{2} .
$$

Now the solutions of Eqs. (4), (6) agree, if

$$
\tilde{W}(t) \sim W(t) \text { for } t \in[0, T]=J,
$$

where $T^{\text {eq }} \leqq T \ll T^{r}, T^{\text {eq }}$ being the relaxation time defined in Appendix B, $T^{r}$ the recurrence time.

If the solutions do agree, we write in a short notation:

$$
\tilde{W} \triangleq W .
$$

It is crucial that this equivalence only can be demanded for the finite time interval $J$. 


\section{An Operator Identity}

In order to investigate the relations between the solutions we introduce first some abbreviations and definitions. Let us choose $W(0)=\tilde{W}(0)$. Then we write:

1. $\tilde{W}-W=\mathbf{N}(t) W(0)$,

2. $\int_{0}^{t} \boldsymbol{K}(\tau) e^{\boldsymbol{M} \tau} \mathrm{d} \tau=\boldsymbol{R}(t)$,

3. $\int_{0}^{t} e^{-\boldsymbol{M} \tau} \mathrm{d} \tau=\boldsymbol{X}(t)$.

We consider the solution of Eq. (4):

$$
\tilde{W}=e^{-\boldsymbol{M} t} W(0)
$$

and insert it into Equation (6). Then we get:

$$
\begin{aligned}
\dot{W}=[ & -\int_{0}^{t} \boldsymbol{K}(t-\tau) e^{-\boldsymbol{M} \tau} \mathrm{d} \tau \\
& \left.+\int_{0}^{t} \boldsymbol{K}(t-\tau) \mathbf{N}(\tau) \mathrm{d} \tau\right] W(0) .
\end{aligned}
$$

After integrating this equation we get with the definitions (9):

$$
\begin{aligned}
W(t) & -W(0) \\
= & -\left[\boldsymbol{R}(t) \boldsymbol{X}(t)-\int_{0}^{t} \boldsymbol{K}(\tau) e^{\boldsymbol{M} \tau} \boldsymbol{X}(\tau) \mathrm{d} \tau\right] W(0) \\
& +\int_{0}^{t} \mathrm{~d} \tau \int_{0}^{\tau} \boldsymbol{K}\left(\tau-\tau^{\prime}\right) \mathbf{N}\left(\tau^{\prime}\right) \mathrm{d} \tau^{\prime} W(0) .
\end{aligned}
$$

Let be $\mathfrak{M}$ the space spanned by the eigenvectors $O_{i}$ with $\lambda_{i} \neq 0$. Hence we have $W(0)=\lambda W^{\text {eq }}+W^{\prime}(0)$ and $W^{\prime}(0) \in \mathfrak{M}$ and therefore $\lambda=1$. Then we get:

$$
\begin{aligned}
& \tilde{W}(t)=W^{\prime}(0)+W^{\mathrm{eq}}+\boldsymbol{N}(t)\left(W^{\prime}(0)+W^{\mathrm{eq}}\right) \\
& \quad+\int_{0}^{t} \mathrm{~d} \tau \int_{0}^{\tau} \boldsymbol{K}\left(\tau-\tau^{\prime}\right) \mathbf{N}\left(\tau^{\prime}\right) \mathrm{d} \tau^{\prime}\left(W^{\prime}(0)+W^{\mathrm{eq}}\right) \\
& \quad-\left[\boldsymbol{R}(t) \boldsymbol{X}(t)-\int_{0}^{t} \boldsymbol{K}(\tau) e^{\boldsymbol{M} \tau} \boldsymbol{X}(\tau) \mathrm{d} \tau\right]\left(W^{\prime}(0)+W^{\mathrm{eq}}\right) .
\end{aligned}
$$

Now we have

$$
\begin{aligned}
& \boldsymbol{R}(t) W^{\mathrm{eq}}=0, \quad \boldsymbol{X}(t) W^{\mathrm{eq}}=t W^{\mathrm{eq}}, \\
& \boldsymbol{N}(t) W^{\mathrm{eq}}=0 .
\end{aligned}
$$

Thus we get:

$$
\begin{gathered}
{\left[1+\boldsymbol{N}(t)+\int_{0}^{t} \mathrm{~d} \tau \int_{0}^{\tau} \mathrm{d} \tau^{\prime} \boldsymbol{K}\left(\tau-\tau^{\prime}\right) \boldsymbol{N}\left(\tau^{\prime}\right) \mathrm{d} \tau^{\prime}\right.} \\
\left.-\boldsymbol{R}(t) \boldsymbol{X}(t)+\int_{0}^{t} \boldsymbol{K}(\tau) e^{\boldsymbol{M} \tau} \boldsymbol{X}(\tau) \mathrm{d} \tau\right] W^{\prime}(0) \\
=e^{-\boldsymbol{M} t} W^{\prime}(0)
\end{gathered}
$$

Now $\boldsymbol{M}$ can be inverted on $\mathfrak{M}$. Thus we get with $\boldsymbol{X}(t)=\left(\mathbf{1}-e^{-\boldsymbol{M} t}\right) \boldsymbol{M}^{-1}$ and the definition (9):

$$
\begin{aligned}
& {\left[1+\boldsymbol{N}(t)+\int_{0}^{t} \mathrm{~d} \tau \int_{0}^{\tau} \mathrm{d} \tau^{\prime} \boldsymbol{K}\left(\tau-\tau^{\prime}\right) \mathbf{N}\left(\tau^{\prime}\right)\right.} \\
& \left.\quad+\boldsymbol{R}(t) e^{-\boldsymbol{M} t} \boldsymbol{M}^{-1}-\int_{0}^{t} \boldsymbol{K}(\tau) \boldsymbol{M}^{-1} \mathrm{~d} \tau-e^{-\boldsymbol{M} t}\right] W^{\prime}(0) \\
& \quad=0 .
\end{aligned}
$$

This identity is valid for all vectors $\in \mathfrak{M}$ (Appen$\operatorname{dix} \mathrm{C}$ ). Then, using that $O \in \mathfrak{M} \Rightarrow \boldsymbol{M} O \in \mathfrak{M}$ we get the following identity on $\mathfrak{R}$ :

$$
\begin{aligned}
\boldsymbol{M} & +\boldsymbol{N}(t) \boldsymbol{M}+\int_{0}^{t} \mathrm{~d} \tau \int_{0}^{\tau} \boldsymbol{K}\left(\tau-\tau^{\prime}\right) \boldsymbol{N}\left(\tau^{\prime}\right) \boldsymbol{M} \\
& +\boldsymbol{R}(t) e^{-\boldsymbol{M} t}-\int_{0}^{t} \boldsymbol{K}(\tau) \mathrm{d} \tau-e^{-\boldsymbol{M} t} \boldsymbol{M} \\
& =\mathbf{0} .
\end{aligned}
$$

If $\mathbf{N}(t)=\mathbf{0}-$ we already mentioned, that this cannot be perfectly true because of the recurrence theorem - we get after a short calculation:

or

$$
\boldsymbol{M}=\int_{0}^{t} \boldsymbol{K}(\tau) \mathrm{d} \tau
$$

$$
\int_{\tau^{\prime}}^{t} K(\tau) \mathrm{d} \tau=0 \text { for } 0<\tau^{\prime} .
$$

Therefore $\boldsymbol{K}(\tau)$ must contain a $\delta$-like singularity:

$\left(\mathrm{F}_{1}\right) \quad \boldsymbol{K}(\tau)=\boldsymbol{M} \delta(\tau)$.

Thus we have

$$
\mathbf{N}(t)=\mathbf{0} \Leftrightarrow\left(\mathrm{F}_{1}\right) .
$$

This result of course can be obtained immediately by comparison of the Laplace transforms of $W, \tilde{W}$, $W=\tilde{W}$. But this argument hides the real physical problem for finite systems. Therefore we must give a careful investigation. Now the question arises in which way $\left(\mathrm{F}_{1}\right)$ must be weakened in order to get physical equivalence of the soultions of the Eqs. (4) and (6).

\section{Conditions of Finite Life-Time and Stability}

Let us investigate some features of Eq. (6). Let be $W_{[0]}(t)$ the solution of Eq. (6) with $W_{[0]}(0)=$ $W(0)$ and $W_{\left[t_{1}\right]}(\tau)$ the solution of Eq. (6) with $W_{\left[t_{1}\right]}(0)=W_{[0]}\left(t_{1}\right)$.

Then we have

$$
W_{\left[t_{1}\right]}(\tau) \neq W_{[0]}\left(t_{1}+\tau\right) .
$$


Proof:

$$
\begin{aligned}
& W_{\left[t_{1}\right]}(\tau)=W_{[0]}\left(t_{1}+\tau\right) \Rightarrow \\
& \partial W_{\left[t_{1}\right]} / \partial \tau=\partial W_{[0]} / \partial t \Rightarrow \\
& -\int_{0}^{\tau} \boldsymbol{K}\left(\tau-\tau_{1}\right) W_{\left[t_{1}\right]}\left(\tau_{1}\right) \mathrm{d} \tau_{1} \\
& \quad=-\int_{0}^{t_{1}+\tau} \boldsymbol{K}\left(t_{1}+\tau-\tau_{1}\right) W_{[0]}\left(\tau_{1}\right) \mathrm{d} \tau_{1} \\
& \Rightarrow \int_{0}^{t_{1}} \boldsymbol{K}\left(t_{1}+\tau-\tau_{1}\right) W_{[0]}\left(\tau_{1}\right) \mathrm{d} \tau_{1}=0
\end{aligned}
$$

for all $\tau$.

This latter relation is wrong. Of course, this result stems from the fact that microscopic information $U-\boldsymbol{G} U$ generates in time - this is the source of the irreversibility. The inequality (14) apparently contradicts the temporal locality - or semigroup property - of Equation (4). We have

$$
\begin{aligned}
\tilde{W}_{\left[t_{1}\right]}(\tau) & =e^{-\boldsymbol{M}\left(t_{1}+\tau\right)} \tilde{W}(0) \\
& =\tilde{W}_{[0]}\left(t_{1}+\tau\right) .
\end{aligned}
$$

This contradiction disappears, if $\left(\mathrm{F}_{1}\right)$ is valid. Let us therefore formulate a weaker condition $\left(\mathrm{F}_{2}\right)$. Before doing this, let us list some relations which are needed as presuppositions in the subsequent analysis. All relations concerning the finite life-time of the kernel $\boldsymbol{K}$ are characterized by (F), all relations concerning closure properties of sets by (C) and all relations concerning the stability of the Eqs. (4) and (6) by (S). We define

$$
\begin{aligned}
& \Sigma=\{W \mid W \in \Re, W(0)=\tilde{W}(0) \Rightarrow W \triangleq \tilde{W}\}, \\
& T=\{V \mid \exists t, W \in \Sigma, W(t)=V \text { or } \tilde{W}(t)=V\} .
\end{aligned}
$$

$\Sigma$ is the set of the "allowed" initial $W, T$ is the set of all $V$, which can occur in equivalent histories. Then we formulate

$$
\left(\mathrm{C}_{1}\right) \quad \Sigma=\{W \mid W \in \Re\} \text {. }
$$

$\left(\mathrm{C}_{1}\right)$ means the validity of the master equation on $\Omega$ without any restrictions.

$\left(\mathrm{F}_{2}\right) \quad$ There is a time $\tau^{*}$ with

$$
V \in T \Rightarrow\left\|\left(\boldsymbol{M}-\int_{0}^{\tau^{*}} \boldsymbol{K}(\tau) \mathrm{d} \tau\right) V\right\| \leqq \varepsilon\|\boldsymbol{M} V\|
$$

and

$$
\left\|\int_{\tau^{*}}^{t} \boldsymbol{K}(\tau) \mathrm{d} \tau V\right\| \leqq \varepsilon\|\boldsymbol{M} V\| \text { for } t \in\left[\tau^{*}, T\right]
$$

Therefore we have:

$$
\left(\mathrm{F}_{2}\right),\left(\mathrm{C}_{1}\right) \Rightarrow\left\|\left(\boldsymbol{M}-\int_{0}^{\tau^{*}} \boldsymbol{K}(\tau) \mathrm{d} \tau\right) W\right\| \leqq \varepsilon\|\boldsymbol{M} W\|,
$$

$$
\left\|\int_{\tau^{*}}^{t} \boldsymbol{K}(\tau) \mathrm{d} \tau W\right\| \leqq \varepsilon\|\boldsymbol{M} W\|
$$

for all $W \in \Re, t \in\left[\tau^{*}, T\right]$,

and, with $X \in \Re \Rightarrow X=\sum \lambda_{i} P_{i}$,

$$
\begin{aligned}
\left(\mathrm{F}_{2}\right),\left(\mathrm{C}_{1}\right) \Rightarrow & \left\|\left(\boldsymbol{M}-\int_{0}^{\tau^{*}} \boldsymbol{K}(\tau) \mathrm{d} \tau\right) \sum \lambda_{i} P_{i}\right\| \\
& \leqq \varepsilon \sum\left|\lambda_{i}\right| d_{i}\left\|\boldsymbol{M} P_{i} / d_{i}\right\| .
\end{aligned}
$$

This relation is discussed in Appendix E. Let us denote the relations

$$
\begin{aligned}
& \left\|\left(\boldsymbol{M}-\int_{0}^{\tau *} \boldsymbol{K}(\tau) \mathrm{d} \tau\right) W\right\| \leqq \varepsilon\|\boldsymbol{M} W\| \\
& \left\|\int_{\tau^{*}}^{t} \boldsymbol{K}(\tau) \mathrm{d} \tau W\right\| \leqq \varepsilon\|\boldsymbol{M} W\|, \quad t \in\left[\tau^{*}, T\right]
\end{aligned}
$$

for all $W$, by $\left(\mathrm{F}_{3}\right)$.

$\left(\mathrm{C}_{2}\right) \quad W \in \Sigma \Rightarrow \tilde{W}(t) \in \Sigma$.

$\left(\mathrm{C}_{3}\right) \quad W \in \Sigma \Rightarrow W(t) \in \Sigma(t)$, where

$\Sigma(t)=\{W(0) \mid W(\tau) \sim \tilde{W}(\tau), \tau \in[0, T-t]\}$.

Now let us turn to some stability conditions

$$
\begin{array}{ll}
\left(\mathrm{S}_{1}\right) \quad & \left\|\tilde{W}_{1}(0)-\tilde{W}_{2}(0)\right\| \leqq \varepsilon \Rightarrow \\
& \left\|\tilde{W}_{1}(t)-\tilde{W}_{2}(t)\right\| \leqq \varepsilon, \quad t \in J . \\
\left(\mathrm{S}_{2}\right) \quad & \left\|W_{1}(0)-W_{2}(0)\right\| \leqq \varepsilon \Rightarrow \\
& \left\|W_{1}(t)-W_{2}(t)\right\| \leqq \varepsilon, \quad t \in J .
\end{array}
$$

$\left(\mathrm{S}_{1}\right),\left(\mathrm{S}_{2}\right)$ mean that Eqs. (4), (6) are stable with respect to small initial disturbaces.

We shall formulate some more relations in the course of our considerations, when we better can understand their physical meaning. Now $\left(\mathrm{F}_{2}\right)$ is a weaker relation than $\left(\mathrm{F}_{1}\right)$. Let us therefore investigate its properties. Let us define

$$
\begin{aligned}
\Omega=\{ & X \mid X \in \mathfrak{R},\left\|\left(\boldsymbol{M}-\int_{0}^{\tau^{*}} \boldsymbol{K}(\tau) \mathrm{d} \tau\right) X\right\| \\
& \leqq \varepsilon\|\boldsymbol{M} X\|,\left\|\int_{\tau^{*}}^{t} \boldsymbol{K}(\tau) \mathrm{d} \tau X\right\| \\
& \left.\leqq \varepsilon\|\boldsymbol{M} X\|, t \in\left[\tau^{*}, t\right]\right\}
\end{aligned}
$$

and let us define

$$
\text { (C) } X \in \Omega \cap \mathfrak{M} \Rightarrow e^{-\boldsymbol{M} t} X, \boldsymbol{M}^{-1} X \in \Omega \cap \mathfrak{M} \text {. }
$$

$\mathfrak{M}$ is defined in Section III.

We define

$$
\int_{0}^{t} \boldsymbol{K}(\tau) \mathrm{d} \tau=\tilde{\boldsymbol{K}}(t) .
$$


Then we get for $t \in\left[\tau^{*}, T\right]$

$$
\begin{aligned}
\boldsymbol{R}(t)= & \tilde{\boldsymbol{K}}(t) e^{\boldsymbol{M} t}-\int_{0}^{t} \mathrm{~d} \tau \tilde{\boldsymbol{K}}(\tau) e^{\boldsymbol{M} \tau} \boldsymbol{M} \\
= & \tilde{\boldsymbol{K}}\left(\tau^{*}\right) e^{\boldsymbol{M} t}+\int_{\tau^{*}}^{t} \boldsymbol{K}(\tau) \mathrm{d} \tau e^{\boldsymbol{M} t} \\
& -\int_{0}^{\tau^{*}} \mathrm{~d} \tau \tilde{\boldsymbol{K}}(\tau) e^{\boldsymbol{M} \tau} \boldsymbol{M}-\int_{\tau^{*}}^{t} \mathrm{~d} \tau \tilde{\boldsymbol{K}}(\tau) e^{\boldsymbol{M} \tau} \boldsymbol{M} .
\end{aligned}
$$

Thus we get from Eq. (11) for $X \in \mathfrak{M}$ :

$$
\begin{aligned}
{[\boldsymbol{N}(t)} & +\int_{0}^{t} \mathrm{~d} \tau \int_{0}^{\tau} \mathrm{d} \tau^{\prime} \boldsymbol{K}\left(\tau-\tau^{\prime}\right) \mathbf{N}\left(\tau^{\prime}\right) \\
& +1-\tilde{\boldsymbol{K}}\left(\tau^{*}\right) \boldsymbol{M}^{-1} \\
& +\int_{\tau^{*}}^{t}\left(\tilde{\boldsymbol{K}}(\tau)-\tilde{\boldsymbol{K}}\left(\tau^{*}\right)\right) e^{-\boldsymbol{M}(t-\tau)} \mathrm{d} \tau \\
& -e^{-\boldsymbol{M} t}+\tilde{\boldsymbol{K}}\left(\tau^{*}\right) \boldsymbol{M}^{-1} e^{-\boldsymbol{M} t} \\
& -\int_{0}^{\tau^{*}} \mathrm{~d} \tau \boldsymbol{K}(\tau) e^{-\boldsymbol{M}^{(t-\tau)} \boldsymbol{M}^{-1}} \\
& \left.+\tilde{\boldsymbol{K}}\left(\tau^{*}\right) e^{-\boldsymbol{M}^{t}} \boldsymbol{M}^{-1}\right] X=0 .
\end{aligned}
$$

The last two terms yield

$$
\begin{aligned}
& {\left[-\int_{0}^{\tau^{*}} \mathrm{~d} \tau \boldsymbol{K}(\tau) e^{-\boldsymbol{M}(t-\tau)} \boldsymbol{M}^{-1}\right.} \\
& \left.+\tilde{\boldsymbol{K}}\left(\tau^{*}\right) e^{-\boldsymbol{M} t} \boldsymbol{M}^{-1}\right] X \\
& =-\int_{0}^{\tau^{*}} \mathrm{~d} \tau \boldsymbol{K}(\tau)\left[e^{\boldsymbol{M} \tau}-1\right] \boldsymbol{M}^{-1} e^{-\boldsymbol{M} t} X .
\end{aligned}
$$

Therefore we have finally

$$
\begin{aligned}
{[\mathbf{N}(t)} & \left.+\int_{0}^{t} \mathrm{~d} \tau \int_{0}^{\tau} \mathrm{d} \tau^{\prime} \boldsymbol{K}\left(\tau-\tau^{\prime}\right) \mathbf{N}\left(\tau^{\prime}\right)\right] X \\
= & {\left[\mathbf{1}-\tilde{\boldsymbol{K}}\left(\tau^{*}\right) \boldsymbol{M}^{-1}+\int_{\tau^{*}}^{t}\left[\tilde{\boldsymbol{K}}(\tau)-\tilde{\boldsymbol{K}}\left(\tau^{*}\right)\right] e^{-\boldsymbol{M}(t-\tau)} \mathrm{d} \tau\right.} \\
& \left.-e^{-\boldsymbol{M} t}+\tilde{\boldsymbol{K}}\left(\tau^{*}\right) \boldsymbol{M}^{-1} e^{-\boldsymbol{M} t}\right] X \\
& -\int_{0}^{\tau^{*}} \mathrm{~d} \tau \boldsymbol{K}(\tau)\left[e^{\boldsymbol{M} \tau}-\mathbf{1}\right] \boldsymbol{M}^{-1} e^{-\boldsymbol{M} t} X
\end{aligned}
$$

Now let be $W^{\prime}(0) \in \Omega-$ therefore $W^{\prime}(0) \in \Omega \cap \mathfrak{M}$. Furthermore we have

$$
\begin{aligned}
& W^{\prime}(0) \in \mathfrak{M} \Rightarrow e^{-\boldsymbol{M} t} W^{\prime}(0) \in \mathfrak{M}, \\
& \boldsymbol{M}^{-1} W^{\prime}(0) \in \mathfrak{M} .
\end{aligned}
$$

$$
\text { If }\left(\mathrm{SM}_{1}\right)\left\|-\int_{0}^{\tau *} \mathrm{~d} \tau \boldsymbol{K}(\tau)\left[e^{\boldsymbol{M} \tau}-1\right] \boldsymbol{M}^{-1} e^{-\boldsymbol{M} t} W^{\prime}(0)\right\| \underset{(29)}{\underset{\varepsilon}{\varepsilon},}
$$

this is the essential smoothness condition on $W^{\prime}(0)$ to be discussed in Part V, then we get

$$
\begin{gathered}
\left\|\left[\mathbf{N}(t)+\int_{0}^{t} \mathrm{~d} \tau \int_{0}^{\tau} \mathrm{d} \tau^{\prime} \boldsymbol{K}\left(\tau-\tau^{\prime}\right) \mathbf{N}\left(\tau^{\prime}\right)\right] W^{\prime}(0)\right\| \\
\leqq \varepsilon\left(\left\|W^{\prime}(0)\right\|+\int_{\tau^{*}}^{t}\left\|e^{-\boldsymbol{M}(t-\tau)} \boldsymbol{M} W^{\prime}(0)\right\| \mathrm{d} \tau\right. \\
\left.+\left\|e^{-\boldsymbol{M} t} W^{\prime}(0)\right\|+1\right) .
\end{gathered}
$$

It can be shown (Appendix F) that the term

$$
\int_{\tau^{*}}^{t}\left\|e^{-\boldsymbol{M}(t-\tau)} \boldsymbol{M} W^{\prime}(0)\right\| \mathrm{d} \tau
$$

remains sufficiently small in time. If the left hand side of (30) is small of order $\varepsilon$, we can, however, not conclude that the single terms are small of order $\varepsilon$. Let us suppose that $\left\|\mathbf{N}(t) W^{\prime}(0)\right\| \gg \varepsilon$.

Then we have from $(30)$

$$
\begin{aligned}
& \tilde{W}^{\prime}(t)-W^{\prime}(0)-\left(W^{\prime}(t)-W^{\prime}(0)\right) \\
& +\int_{0}^{t} \mathrm{~d} \tau \int_{0}^{\tau} \mathrm{d} \tau^{\prime} \boldsymbol{K}\left(\tau-\tau^{\prime}\right)\left(\tilde{W}^{\prime}\left(\tau^{\prime}\right)-W^{\prime}\left(\tau^{\prime}\right)\right)=A(t), \\
& \|A(t)\|=O(\varepsilon) .
\end{aligned}
$$

Thus we get: The integral equation

$$
X(t)-X(0)+\int_{0}^{t} \mathrm{~d} \tau \int_{0}^{\tau} \boldsymbol{K}\left(\tau-\tau^{\prime}\right) X\left(\tau^{\prime}\right) \mathrm{d} \tau^{\prime}=0,
$$

which is equivalent to Eq. (6) is not stable with respect to a small disturbance $A(t)$. If this is the case, that Eq. (6) is without physical sense - there is no experimental test of it. Thus we formulate

$$
\begin{aligned}
& \left\|\left[\mathbf{N}(t)+\int_{0}^{t} \mathrm{~d} \tau \int_{0}^{\tau} \mathrm{d} \tau^{\prime} \mathbf{K}\left(\tau-\tau^{\prime}\right) \mathbf{N}\left(\tau^{\prime}\right)\right] W^{\prime}(0)\right\| \leqq \varepsilon \\
& \Rightarrow\left\|\mathbf{N}(t) W^{\prime}(0)\right\| \leqq \varepsilon,
\end{aligned}
$$

without investigating the mathematical aspect of this relation. Thus we finally have

$$
W^{\prime}(0) \in \Omega, \quad\left(\mathrm{SM}_{1}\right),\left(\mathrm{C}_{4}\right),\left(\mathrm{S}_{3}\right) \Rightarrow \tilde{W} \triangleq W .
$$

If $\Omega=\Re$, we have

$$
\left(\mathrm{SM}_{1}\right),\left(\mathrm{S}_{3}\right) \Rightarrow \tilde{W} \triangleq W .
$$

Now let us investigate the reversion. Let us again start with Eq. (10) and let $\left\|\boldsymbol{N}(t) W^{\prime}(0)\right\| \leqq \varepsilon$. Again we must investigate the expression

$$
\left[\mathbf{N}(t)+\int_{0}^{t} \mathrm{~d} \tau \int_{0}^{\tau} \mathrm{d} \tau^{\prime} \boldsymbol{K}\left(\tau-\tau^{\prime}\right) \mathbf{N}\left(\tau^{\prime}\right)\right] W^{\prime}(0)=F .
$$

We have

$$
\begin{aligned}
F= & \tilde{W}^{\prime}(t)-W^{\prime}(0)-\left(W^{\prime}(t)-W^{\prime}(0)\right) \\
& +\int_{0}^{t} \mathrm{~d} \tau \int_{0}^{\tau} \mathrm{d} \tau^{\prime} \boldsymbol{K}\left(\tau-\tau^{\prime}\right)\left(\tilde{W}^{\prime}\left(\tau^{\prime}\right)-W^{\prime}\left(\tau^{\prime}\right)\right) \\
= & \tilde{W}^{\prime}-W^{\prime}(0)+\int_{0}^{t} \mathrm{~d} \tau \int_{0}^{\tau} \mathrm{d} \tau^{\prime} \boldsymbol{K}\left(\tau-\tau^{\prime}\right) \tilde{W}^{\prime}\left(\tau^{\prime}\right) .
\end{aligned}
$$


If the two histories $\tilde{W}^{\prime}, W^{\prime}$ are physically equivalent and if $\|F(t)\| \gg \varepsilon$, we must conclude that two equivalent histories do not both fulfill the integral equation which defines one of the histories. This again would be without physical sense. Again we do not discuss the mathematical aspect. Instead of that we presuppose

$$
\begin{aligned}
& \left\|\boldsymbol{N}(\tau) W^{\prime}(0)\right\| \leqq \varepsilon, \quad 0 \leqq \tau \leqq t \Rightarrow \\
& \left\|\int_{0}^{t} \mathrm{~d} \tau \int_{0}^{\tau} \mathrm{d} \tau^{\prime} \boldsymbol{K}\left(\tau-\tau^{\prime}\right) \boldsymbol{N}\left(\tau^{\prime}\right) W^{\prime}(0)\right\| \leqq m \varepsilon .
\end{aligned}
$$

We have

$$
\begin{array}{cl} 
& \left\{\|F(t)\| \leqq \varepsilon,\left(\mathrm{S}_{3}\right)\right\} \Rightarrow\left(\mathrm{S}_{4}\right), \\
& \left\{\left\|\mathbf{N}(t) W^{\prime}(0)\right\| \leqq \varepsilon,\left(\mathrm{S}_{4}\right)\right\} \Rightarrow \\
& \left\|F^{\prime}(t)\right\| \leqq(m+1) \varepsilon, \\
\text { or } \quad & \left\{\left\|\mathbf{N}(t) W^{\prime}(0)\right\| \leqq \varepsilon,\left(\mathrm{S}_{4}\right)\right\} \Rightarrow\left(\mathrm{S}_{3}\right) .
\end{array}
$$

Note that $\left(\mathrm{S}_{4}\right)$ becomes trivial for $\left\|\mathbf{N}(t) W^{\prime}(0)\right\| \equiv \mathbf{0}$. Thus we get

$$
\begin{aligned}
& W(0) \in \Sigma,\left(\mathbf{S}_{4}\right) \\
& \Rightarrow \|\left[\mathbf{1}+\boldsymbol{R}(t) e^{-\boldsymbol{M} t} \boldsymbol{M}^{-1}\right. \\
& \left.-\int_{0}^{t} \boldsymbol{K}(\tau) \boldsymbol{M}^{-1} \mathrm{~d} \tau-e^{-\boldsymbol{M} t}\right] W^{\prime}(0) \| \\
& \leqq(m+1) \varepsilon .
\end{aligned}
$$

There is no obvious connection between the halfgroup property (15) and $\left(\mathrm{S}_{4}\right)$. Let us therefore investigate the validity of a weaker halfgroup property of Equation (6).

We want to obtain

$$
W_{\left[t_{1}\right]}(\tau) \sim W_{[0]}\left(t_{1}+\tau\right)
$$

in order to ensure the approximative halfgroup property of Eq. (6). Let be $W(0) \in \Sigma$. Therefore we have

$$
W_{[0]}\left(t_{1}+\tau\right) \sim e^{-\boldsymbol{M}\left(t_{1}+\tau\right)} W(0)=e^{-\boldsymbol{M} \tau} e^{-\boldsymbol{M} t_{1}} W(0)
$$
and

$$
W_{[0]}\left(t_{1}\right)=W_{\left[t_{1}\right]}(0) \sim e^{-\boldsymbol{M} t_{1}} W(0) .
$$

Now let us assume the validity of $\left(\mathrm{C}_{2}\right),\left(\mathrm{S}_{2}\right)((21)$, (24)). Then we get $W_{\left[t_{1}\right]}(\tau) \sim V_{\left[t_{1}\right]}(\tau)$ where $V_{\left[t_{1}\right]}(\tau)$ is the solution of Eq. (6) with $V_{\left[t_{1}\right]}(0)=e^{-\boldsymbol{M} t_{1}} W(0)$. Thus we get with $\left(\mathrm{C}_{2}\right)$ :

$$
\begin{gathered}
V_{\left[t_{1}\right]}(\tau) \sim e^{-\boldsymbol{M} \tau} e^{-\boldsymbol{M} t_{1}} W(0), \quad \text { or } \\
\left\|W_{\left[t_{1}\right]}(\tau)-W_{[0]}\left(t_{1}+\tau\right)\right\| \leqq 3 \varepsilon .
\end{gathered}
$$

Therefore we get by use of

$$
W(t)-W(0)=-\int_{0}^{t} \mathrm{~d} \tau \int_{0}^{\tau} \mathrm{d} \tau^{\prime} \boldsymbol{K}\left(\tau-\tau^{\prime}\right) W\left(\tau^{\prime}\right)
$$

after some calculation

$$
\begin{aligned}
& \| \int_{0}^{\tau} \mathrm{d} \tau^{\prime} \int_{-t_{1}}^{0} \boldsymbol{K}\left(\tau^{\prime}-\tau^{\prime \prime}\right) W_{[0]}\left(t_{1}+\tau^{\prime \prime}\right) \mathrm{d} \tau^{\prime \prime} \\
& +\int_{0}^{\tau} \mathrm{d} \tau^{\prime} \int_{0}^{\tau^{\prime}} \boldsymbol{K}\left(\tau^{\prime}-\tau^{\prime \prime}\right) \\
& \cdot\left[W_{\left[t_{1}\right]}\left(\tau^{\prime \prime}\right)-W_{[0]}\left(t_{1}+\tau^{\prime \prime}\right)\right] \mathrm{d} \tau^{\prime \prime} \| \leqq 3 \varepsilon .
\end{aligned}
$$

This latter relation implies some properties of the kind $(\mathrm{F})$ provided, that there are stability properties of the kind (S) concerning the second term. We must, however, use additional assumptions in order to get $\left(\mathrm{F}_{2}\right)$. Let us therefore return to $(33)$. Let us assume after a time $\tau^{*}$ not only $W(t) \sim \tilde{W}(t)$ but also $\dot{W} \sim \dot{\tilde{W}}$. That means, together with a stability property:

(D) $\left\|\left[\boldsymbol{R}\left(t+\tau^{*}\right) e^{-\boldsymbol{M}\left(t+\tau^{*}\right)}-\boldsymbol{M} e^{-\boldsymbol{M}\left(t+\tau^{*}\right)}\right] W^{\prime}(0)\right\|$

$$
\begin{aligned}
& \leqq \varepsilon\left\|\boldsymbol{M} \tilde{W}^{\prime}\left(t+\tau^{*}\right)\right\|, \\
& W(0) \in \Sigma, \quad t \geqq 0 .
\end{aligned}
$$

We demand this property to be valid for

$\Sigma^{\prime}=\left\{\boldsymbol{M}^{-1} W^{\prime}(0), W(0)=W^{\prime}(0)+W\right.$ eq $\left.\varepsilon \Sigma\right\}$.

Thus we get

$$
\begin{array}{r}
\left\|\boldsymbol{R}(t) e^{-\boldsymbol{M} t} \boldsymbol{M}^{-1} W^{\prime}(0)-e^{-\boldsymbol{M} t} W^{\prime}(0)\right\| \\
\leqq \varepsilon\left\|e^{-\boldsymbol{M} t} W^{\prime}(0)\right\|,
\end{array}
$$

and thus

$$
\begin{aligned}
& \left\|\left[\mathbf{1}-\int_{0}^{t} \boldsymbol{K}(\tau) \boldsymbol{M}^{-1} \mathrm{~d} \tau\right] W^{\prime}(0)\right\| \\
& \quad \leqq(m+1) \varepsilon+\varepsilon\left\|\tilde{W}^{\prime}(t)\right\|=O(\varepsilon) .
\end{aligned}
$$

Hence we have the following result:

$$
\left(\mathrm{S}_{4}\right), W(0) \in \Sigma, \quad(\mathrm{D}) \text { valid on } \Sigma^{\prime} \Rightarrow
$$

and

$$
\left\|\left[1-\int_{0}^{\tau^{*}} \boldsymbol{K}(\tau) \boldsymbol{M}^{-1}\right] W^{\prime}(0)\right\|=O(\varepsilon)
$$

where

$$
\left\|\int_{\tau^{*}}^{\tau^{*}+t} \boldsymbol{K}(\tau) \boldsymbol{M}^{-1} W^{\prime}(0)\right\|=O(\varepsilon)
$$

or

$$
W(0)=W^{\prime}(0)+W^{\text {eq }} \in \Sigma, \quad 0 \leqq t \leqq T
$$

$$
\boldsymbol{M}=\int_{0}^{\tau^{*}} \boldsymbol{K}(\tau) \mathrm{d} \tau, \quad \boldsymbol{K}(\tau)=0, \quad \tau \geqq \tau^{*},
$$

as a more striking - but more unprecise - formulation.

Let us consider the reversion

$$
\begin{aligned}
& \left(\mathrm{S}_{4}\right), W(0) \in \Sigma, \\
& \left\|\left[1-\int_{0}^{\tau^{*}} \boldsymbol{K}(\tau) \boldsymbol{M}^{-1} \mathrm{~d} \tau\right] W^{\prime}(0)\right\|=O(\varepsilon),
\end{aligned}
$$




$$
\begin{aligned}
\left\|\int_{\tau^{*}}^{\tau^{*}+t} \boldsymbol{K}(\tau) \boldsymbol{M}^{-1} W^{\prime}(0)\right\|=O(\varepsilon) \Rightarrow & \\
\left\|\left[\boldsymbol{R}(t) e^{-\boldsymbol{M} t} \boldsymbol{M}^{-1}-e^{-\boldsymbol{M} t}\right] W^{\prime}(0)\right\| & =O(\varepsilon), \\
t & \geqq \tau^{*} .
\end{aligned}
$$

This corresponds to (D).

Let us now give a remark. We assume for the moment that $\|\mathbf{K}(t) W(0)\|=O(\varepsilon)$ for all $W(0)$ and $\tau^{*} \leqq t \leqq T$ eq. That is stronger than Equation (38). On the other hand we have from Eq. (6), putting

$$
\exp [i(\mathbf{1}-\boldsymbol{G}) \boldsymbol{L}(\mathbf{1}-\boldsymbol{G}) t]=\boldsymbol{V}(t),
$$

$$
\begin{aligned}
\int_{0}^{\tau^{*}} K(\tau) \mathrm{d} \tau= & i \boldsymbol{G L}(\mathbf{1}-\boldsymbol{G})[(1-G) L(1-G)]^{-1} \\
& \cdot(\mathbf{1}-\boldsymbol{G}) \boldsymbol{L} \boldsymbol{G}-i \boldsymbol{G L}(\mathbf{1}-\boldsymbol{G}) \boldsymbol{V}\left(\tau^{*}\right) \\
& \cdot[(\mathbf{1}-\boldsymbol{G}) \boldsymbol{L}(\mathbf{1}-\boldsymbol{G})]^{-1}(\mathbf{1}-\boldsymbol{G}) \boldsymbol{L} \boldsymbol{G} \\
\cong & M .
\end{aligned}
$$

Now the eigenvalues of $\boldsymbol{M}$ cannot be pure imaginary for reasons of irreversibility. But the operator

$$
G L(1-G)[(1-G) L(1-G)]^{-1}(1-G) L G
$$

is selfadjoint. Hence we must conclude that the operator

$$
-i \boldsymbol{G L}(\mathbf{1}-\boldsymbol{G}) \boldsymbol{V}\left(\tau^{*}\right)[(\mathbf{1}-\boldsymbol{G}) \boldsymbol{L}(\mathbf{1}-\boldsymbol{G})]^{-1}
$$

$$
\cdot(\mathbf{1}-\boldsymbol{G}) \boldsymbol{L} \boldsymbol{G}
$$

cannot be neglected.

If the equation

$$
\begin{gathered}
{[(1-G) L(1-G)]^{-1} V\left(\tau^{*}\right)(1-G) L G W} \\
=V(t)(1-G) L G W
\end{gathered}
$$

has a nontrivial solution, $W$ being a statistical operator, then we get

$$
\begin{aligned}
- & i \boldsymbol{G L}(\mathbf{1}-\boldsymbol{G}) \boldsymbol{V}\left(\tau^{*}\right) \\
& \cdot[(\mathbf{1}-\boldsymbol{G}) \boldsymbol{L}(\mathbf{1}-\boldsymbol{G})]^{-1}(\mathbf{1}-\boldsymbol{G}) \boldsymbol{L} \boldsymbol{G} W \\
= & -i \boldsymbol{G L}(\mathbf{1}-\boldsymbol{G}) \boldsymbol{V}(t)(\mathbf{1}-\boldsymbol{G}) \boldsymbol{L} \boldsymbol{G} W \\
= & O(\varepsilon)
\end{aligned}
$$

after presupposition. That means

$$
\begin{aligned}
\int_{0}^{\tau^{*}} \boldsymbol{K}(\tau) \mathrm{d} \tau W \cong & i \boldsymbol{G L}(\mathbf{1}-\boldsymbol{G})[(\mathbf{1}-\boldsymbol{G}) L(\mathbf{1}-\boldsymbol{G})]^{-1} \\
& \cdot(\mathbf{1}-\boldsymbol{G}) \boldsymbol{L} \boldsymbol{G} W \\
= & i \boldsymbol{S} W,
\end{aligned}
$$

where $\boldsymbol{S}$ is selfadjoint. Hence, by putting

$$
\int_{0}^{\tau^{*}} \boldsymbol{K}(\tau) \mathrm{d} \tau W=i \boldsymbol{S} W,
$$

we would be led to a solution without tend to equilibrium. This conclusion of course depends on the magnitude of the nonhermitian part $\boldsymbol{M}^{\prime}$ of $\boldsymbol{M}$. We have assumed that $\left\|\boldsymbol{M}^{\prime} W(0)\right\| \gg O(\varepsilon)$. Therefore we may conclude: The equation (40) is not allowed to have a nontrivial solution. This corresponds in a certain sense to a condition of nonergodicity. In fact there must be some $Z \in \mathcal{L} \ominus \Omega$, which cannot be reached by any

$$
\begin{aligned}
& \boldsymbol{V}(t)(\mathbf{1}-\boldsymbol{G}) \boldsymbol{L} \boldsymbol{G} W, \quad \tau^{*} \leqq t \leqq T \mathrm{eq}, \\
& Z=[(\mathbf{1}-\boldsymbol{G}) \boldsymbol{L}(\mathbf{1}-\boldsymbol{G})]^{-1} \boldsymbol{V}\left(\tau^{*}\right)(\mathbf{1}-\boldsymbol{G}) \boldsymbol{L} \boldsymbol{G} W .
\end{aligned}
$$

\section{Smoothing in Time}

Let us consider Eq. (6)

$$
\dot{V}=-\int_{0}^{t} \boldsymbol{K}(t-\tau) \boldsymbol{V}(\tau) \mathrm{d} \tau, \quad V=W-W^{\text {eq }} .
$$

Let us "renormalize" the kernel $\boldsymbol{K}: \boldsymbol{K}(t)=0$ for $t \geqq T$ eq. Then we define the temporal smoothing $\boldsymbol{Q}[\Omega]$ by

$$
\begin{aligned}
Q[\Omega] V & =V_{1}(\Omega, t)=\frac{1}{\sqrt{2 \pi}} \int_{-\Omega}^{+\Omega} \hat{V}(\omega) e^{-i \omega t} \mathrm{~d} \omega, \\
\hat{V}(\omega) & =\frac{1}{\sqrt{2} \pi} \int_{0}^{\infty} V(t) e^{i \omega t} \mathrm{~d} t .
\end{aligned}
$$

We put $V(t)=V_{1}(t)+V_{2}(t)$.

After a short calculation we have

$$
\dot{V}_{1}=Q[\Omega] \dot{V}+(\sin \Omega t / \pi t) V(0) .
$$

We look for an equation for $V_{1}$, hence we must consider $\boldsymbol{Q}[\Omega] \dot{V}$. We have

$$
\begin{aligned}
\dot{V}= & -\int_{0}^{t} \boldsymbol{K}(t-\tau) V_{1}(\tau) \mathrm{d} \tau \\
& -\int_{0}^{t} \boldsymbol{K}(t-\tau) V_{2}(\tau) \mathrm{d} \tau .
\end{aligned}
$$

From the definition of $\boldsymbol{Q}[\Omega]$ it follows that

$$
\boldsymbol{Q}[\Omega] \int_{0}^{t} \boldsymbol{K}(t-\tau) V_{2}(\tau) \mathrm{d} \tau \equiv 0 .
$$

Thus we are left with

$$
\boldsymbol{Q}[\Omega] \dot{V}=-\boldsymbol{Q}[\Omega] \int_{0}^{t} \boldsymbol{K}(t-\tau) V_{1}(\tau) \mathrm{d} \tau .
$$

Now we have

$$
\begin{aligned}
\int_{0}^{t} \boldsymbol{K}(t-\tau) V_{1}(\tau) \mathrm{d} \tau= & \int_{0}^{\tau^{*}} \boldsymbol{K}(\tau) \mathrm{d} \tau V_{1}(t-\tau) \\
& +\int_{\tau^{*}}^{t} \boldsymbol{K}(\tau) \mathrm{d} \tau V_{1}(t-\tau) .
\end{aligned}
$$


We now assume the validity of $\left(\mathrm{F}_{3}\right)(20)$. Then we have a first condition for $\Omega$. $\Omega$ must be chosen so small that

$$
\begin{aligned}
& \| \int_{0}^{\tau^{*}} \boldsymbol{K}(\tau) \mathrm{d} \tau V_{1}(t-\tau) \\
& \quad-\int_{0}^{\tau^{*}} \boldsymbol{K}(\tau) \mathrm{d} \tau V_{1}(t) \|=O(\varepsilon) \\
& \| \int_{\tau^{*}}^{t} \boldsymbol{K}(\tau) \mathrm{d} \tau V_{1}(t-\tau) \\
& \quad-\int_{\tau^{*}}^{t} \boldsymbol{K}(\tau) \mathrm{d} \tau V_{1}(t) \|=O(\varepsilon) .
\end{aligned}
$$

Hence we get from $\left(\mathrm{F}_{3}\right)$

$$
\begin{aligned}
& \int_{0}^{t} \boldsymbol{K}(t-\tau) V_{1}(\tau) \mathrm{d} \tau \\
& =\chi(t) D(t)+\int_{0}^{\tau^{*}} \boldsymbol{K}(\tau) \mathrm{d} \tau V_{1}(t)+O(\varepsilon),
\end{aligned}
$$

where

$$
\begin{aligned}
\chi(t) & =\left\{\begin{array}{ll}
1, & 0 \leqq t \leqq \tau^{*} \\
0, & \text { else }
\end{array}\right\}, \\
D(t) & =\int_{0}^{t} \boldsymbol{K}(t-\tau) V_{1}(\tau) \mathrm{d} \tau-\int_{0}^{\tau^{*}} \boldsymbol{K}(\tau) \mathrm{d} \tau V_{1}(t) .
\end{aligned}
$$

Thus we get with $\int_{0}^{\tau *} \boldsymbol{K}(\tau) \mathrm{d} \tau=\boldsymbol{M}$ :

$$
\boldsymbol{Q}[\Omega] \dot{\boldsymbol{V}}=-\boldsymbol{M} V_{1}-\boldsymbol{Q}[\Omega](\chi D)+O(\varepsilon)
$$

Now we impose a second condition on $\Omega$ :

$\left(\mathrm{SM}_{3}\right) \quad \boldsymbol{Q}[\Omega](\chi D)=O(\varepsilon)$.

We get

$$
\begin{aligned}
& \widehat{\chi D}(\omega)=\frac{1}{V^{2} \pi} \int_{0}^{\tau *} D(\tau) e^{i \omega \tau} \mathrm{d} \tau \Rightarrow \\
& \boldsymbol{Q}[\Omega](\chi D)=\frac{1}{\pi} \int_{0}^{\tau^{*}} D(\tau) \frac{\sin \Omega(t-\tau)}{t-\tau} \mathrm{d} \tau .
\end{aligned}
$$

Thus we have

$$
\|\boldsymbol{Q}[\Omega](\chi D)\| \leqq \Omega / \pi \operatorname{Max}\|D(\tau)\| \tau^{*},
$$

and $\left(\mathrm{SM}_{2}\right)$ is fulfilled, if

$$
\frac{\Omega \tau^{*}}{\pi} \underset{\left[0, \tau^{*}\right]}{\operatorname{Max}}\|D(\tau)\| \leqq \varepsilon .
$$

Hence we get from Eq. (42)

$$
\begin{aligned}
\dot{V}_{1}= & -\boldsymbol{M} V_{1}+\frac{\sin \Omega t}{\pi t} V(0)+O(\varepsilon) \\
= & -\boldsymbol{M} V_{1}+\chi(t) \frac{\sin \Omega t}{\pi t} V(0) \\
& +(1-\chi(t)) \frac{\sin \Omega t}{\pi t} V(0) .
\end{aligned}
$$

Our third condition on $\Omega$ reads

$\left(\mathrm{SM}_{4}\right) \quad \sin \Omega \tau^{*} / \pi \tau^{*} \leqq \varepsilon$,

yielding

$$
\dot{V}_{1}=-\boldsymbol{M} V_{1}+\chi(t) \frac{\sin \Omega t}{\pi t} V(0)+O(\varepsilon)(t) .
$$

$\left(\mathrm{S}_{5}\right)$ Now we must assume that Eq. (4) is stable with respect to the disturbance

$$
O(\varepsilon)(t) .
$$

Hence we get

$$
\begin{aligned}
V_{1}(\Omega, t)= & e^{-\boldsymbol{M} t}\left[V_{1}(0)+\frac{1}{\pi} \int_{0}^{\tau^{*}} e^{\boldsymbol{M} \tau} \frac{\sin \Omega \tau}{\tau} V(0)\right] \\
& +O(\varepsilon), \quad t \geqq \tau^{*} .
\end{aligned}
$$

Furthermore we have

$$
V_{1}(\Omega, 0)=\frac{1}{\pi} \int_{0}^{\infty} V(t) \frac{\sin \Omega t}{t} \mathrm{~d} t .
$$

With (45) we get

$$
V_{1}(\Omega, 0)=\frac{1}{\pi} \int_{0}^{\tau^{*}} V(t) \frac{\sin \Omega t}{t} \mathrm{~d} t+O(\varepsilon),
$$

yielding

$$
\begin{aligned}
V_{1}(\Omega, t)= & e^{-\boldsymbol{M} t}\left[\frac{1}{\pi} \int_{0}^{\tau^{*}} \mathrm{~d} \tau \frac{\sin \Omega \tau}{\tau}\left(V(\tau)+e^{\boldsymbol{M} \tau} V(0)\right)\right] \\
& +O(\varepsilon), \quad t \geqq \tau^{*} .
\end{aligned}
$$

This means: The smooth part $V_{1}(\Omega, t)$ of $V$ obeys a master equation $\dot{V}_{1}=-\boldsymbol{M} V_{1}$ for $t \geqq \tau^{*}$.

$V_{1}\left(\tau^{*}\right)$ is given by

$$
V_{1}\left(\tau^{*}\right)=e^{-\boldsymbol{M} \tau^{*}}\left[\frac{1}{\pi} \int_{0}^{\tau^{*}} \mathrm{~d} \tau \frac{\sin \Omega \tau}{\tau}\left(V(\tau)+e^{\boldsymbol{M} \tau} V(0)\right)\right] .
$$

Note, that $\Omega$ must fulfill several conditions. Now we have $V_{1}(t)=V(t)+O(\varepsilon)$, if $V(0)$ is spanned by slowly varying operators $A_{j}: \boldsymbol{Q}[\Omega] A_{j}(t) \cong A_{j}(t)$. This result of course is closely related to those in [5], [6]. If the solution $W$ of

$$
\dot{W}=-\int_{0}^{t} \boldsymbol{K}(t-\tau) W(\tau) \mathrm{d} \tau
$$

is smooth in intervals of length $\tau^{*}$,then it agrees with $\boldsymbol{Q}[\Omega] W . \boldsymbol{Q}[\Omega] W$ obeys a master equation for $t \geqq \tau^{*}$.

\section{Conclusions and Discussion}

The simple argument which yields complete agreement between the solution of the master 
equation and the exact equation is

$$
\left(\mathrm{F}_{1}\right): \boldsymbol{K}(\tau)=\boldsymbol{M} \delta(\tau) .
$$

Of course, $\left(\mathrm{F}_{1}\right)$ cannot be valid exactly. Hence we look for a weaker form $\left(\mathrm{F}_{2}\right)$, hoping that

$$
\tilde{W} \triangleq W \Leftrightarrow\left(\mathrm{F}_{2}\right) \text {. }
$$

We have seen that additional assumptions must be made in order to get such a result. First of all we must have in mind that the master equation possibly is not valid for all $W$. Hence we must introduce assumptions concerning the set of the allowed initial $W(0)$. Given relations of the kind $(\mathrm{F})$ defining a set $\Omega(25)$, we must demand :

1. an additional smoothness property $\left(\mathrm{SM}_{1}\right)$ for $W(0)(29)$,

2. a closure property $\left(\mathrm{C}_{4}\right)$ for $\Omega$ and

3. a stability property $\left(\mathrm{S}_{3}\right)$.

Then we have (32):

$$
\left(\mathrm{SM}_{1}\right), W(0)-W^{\mathrm{eq}} \in \Omega,\left(\mathrm{C}_{4}\right),\left(\mathrm{S}_{3}\right) \Rightarrow \tilde{W} \triangleq W
$$

Conversely we have (38):

$$
\begin{aligned}
& \tilde{W} \triangleq W,\left(\mathrm{~S}_{4}\right),\left(\mathrm{C}_{4}\right), D \text { valid on } \boldsymbol{M}^{-1}(\Sigma) \\
& \Rightarrow \boldsymbol{M}^{-1} W^{\prime}(0) \in \Omega .
\end{aligned}
$$

Furthermore we have

$$
\begin{aligned}
& \left(\mathrm{S}_{4}\right), W(0) \in \Sigma, \quad \boldsymbol{M}^{-1} W^{\prime}(0) \in \Omega \\
& \Rightarrow D \text { valid on } \boldsymbol{M}^{-1}(\Sigma),
\end{aligned}
$$

and from Section $\mathrm{V}$

$$
\left(\mathrm{SM}_{2}\right),\left(\mathrm{SM}_{3}\right),\left(\mathrm{SM}_{4}\right),\left(\mathrm{S}_{5}\right), \mathrm{F}_{3} \Rightarrow \tilde{W} \hat{=} \hat{W} \text {. }
$$

Let us discuss these relations. If $W(0)$ is spanned by operators which vary slowly in time and if $W^{\prime}(0) \in \Omega$, which means that

$$
\boldsymbol{M} W^{\prime}(0) \sim \int_{0}^{\tau *} \boldsymbol{K}(\tau) \mathrm{d} \tau W^{\prime}(0)
$$

and if $\Omega$ is closed $\left(\mathrm{C}_{4}\right)$ and if the stability property (S3) holds, then $\tilde{W} \triangleq W$. Conversely: If $\tilde{W} \triangleq W$, then under the condition $\left(\mathrm{S}_{4}\right)$ and the equivalence of the equations of motion (not only of their solutions), $W^{\prime}(0) \in \Omega$. It turns out that this latter property is equivalent to the equivalence of the equations, if $\left(\mathrm{S}_{4}\right)$ is valid and $W(0) \in \Sigma$, or: If the stability property $\left(\mathrm{S}_{4}\right)$ is valid and if $\tilde{W} \hat{}$ W, then the equivalence of the equations for $t \geqq \tau^{*}$ is equivalent to

$$
\boldsymbol{M} W^{\prime}(0) \cong \int_{0}^{\tau *} \boldsymbol{K}(\tau) \mathrm{d} \tau W^{\prime}(0) .
$$

Regarding the result of Section V, we see that there is another set of presuppositions which yield $\tilde{W} \triangleq W$. But we see again that we must use smoothness conditions, conditions of finite life-time and conditions of stability.

Of course, we are not sure that the sufficient conditions for the proofs are necessary, too. The stability properties seem to be weak, if these stability properties would not hold, all our equations would be without physical sense. We must have in mind that these equations govern the behaviour of ensembles and not the behaviour of single members of these ensembles. Hence turbulence-like phenomena can - and must - be described by stable equations of motion for the corresponding ensembles. Our considerations are simplified, if the master equation is assumed to be valid for all initial $W(0)$. This is of interest with respect to critical phenomena occuring in special energy shells. It is beyond the aim of this paper to investigate this question.

\section{Appendices}

\section{A. Proof of: $\boldsymbol{G U}>0$}

We have $\boldsymbol{G} U=\sum \lambda_{i} P_{i}$. Hence

$$
\left(P_{j} \boldsymbol{G} U\right)=\left(G P_{j} ; U\right)=\left(P_{j} ; U\right) \geqq 0
$$

and $\lambda_{j}=\left(\boldsymbol{G} U ; P_{j}\right) / d_{j} \geqq 0$.

\section{B. Definition of $T$ eq}

We define

$$
\begin{aligned}
& T^{\mathrm{eq}}=\inf \{T \mid t>T \\
& \left.\quad \Rightarrow\left\|e^{-\boldsymbol{M} t} W(0)-W^{\mathrm{eq}}\right\| \leqq \varepsilon \text { for all } W(0)\right\} .
\end{aligned}
$$

Thus

$$
T^{\mathrm{eq}}=\inf \left\{T \mid t>T \Rightarrow\left\|e^{-M t} W^{\prime}(0)\right\| \leqq \varepsilon\right\} .
$$

Now we have $W^{\prime}(0)=\sum \gamma_{i} O_{i}(5)$, therefore

$$
\left\|e^{-\boldsymbol{M} t} W^{\prime}(0)\right\|^{2}=\sum e^{-\left(\lambda_{i}^{*}+\lambda_{j}\right) t} \gamma_{i} *\left(O_{i} ; O_{j}\right) \text {. }
$$

This expression is difficult, thus we simplify:

$$
\left\|e^{-\boldsymbol{M} t} W^{\prime}(0)\right\| \leqq \sum\left|\gamma_{i}\right| e^{-r_{i} t},
$$

because $\left\|O_{i}\right\|=1, \lambda_{i}=r_{i}+i s_{i}$. Now we have

$$
\left\|e^{-\boldsymbol{M} t} W^{\prime}(0)\right\|^{2} \leqq \sum\left|\gamma_{i}\right|^{2} \sum e^{-2 r_{i} t} .
$$

On the other hand:

$$
\begin{aligned}
\left\|W^{\prime}(0)\right\|^{2} & =\sum \gamma_{i}{ }^{*} \gamma_{j}\left(O_{i} ; O_{j}\right) \\
& =\sum \gamma_{i} * \gamma_{j} \Omega_{i j}=(\gamma|\Omega| \gamma) .
\end{aligned}
$$


$\Omega$ is selfadjoint, hence $(\gamma|\Omega| \gamma) \geqq \boldsymbol{\omega} \sum\left|\gamma_{i}\right|^{2}$. Thus we get

$$
\sum\left|\gamma_{i}\right|^{2} \leqq\left\|W^{\prime}(0)\right\|^{2} / \omega,
$$

$\boldsymbol{\omega}$ being the minimal eigenvalue of $\Omega, \boldsymbol{\omega}>0$. Therefore we get

$$
\left\|e^{-\boldsymbol{M} t} W^{\prime}(0)\right\|^{2} \leqq\left\{\left\|W^{\prime}(0)\right\|^{2} / \boldsymbol{\omega}\right\} \varrho e^{-2 \boldsymbol{r} t},
$$

where $\varrho=\operatorname{dim} \mathfrak{R}, \boldsymbol{r}=\min r_{i}$.

Now $\left\|W^{\prime}(0)\right\|^{2} \leqq 1 / \boldsymbol{d}-1 / d$ with $\quad \boldsymbol{d}=\min d_{i}$ (Appendix D). Hence we have

$$
\left\|e^{-\boldsymbol{M} t} W^{\prime}(0)\right\|^{2} \leqq(\varrho / \boldsymbol{\omega} \boldsymbol{d}) e^{-2 \boldsymbol{r} t} .
$$

Therefore we get

$$
(\varrho / \omega \boldsymbol{d}) e^{-2 \boldsymbol{r} t} \leqq \varepsilon^{2} \Rightarrow t>T^{\mathrm{eq}}
$$

or

$$
\begin{aligned}
T \mathrm{eq} & \leqq-\frac{\log \varepsilon}{\boldsymbol{r}}+\frac{1}{\boldsymbol{r}} \log (\varrho / \boldsymbol{\omega} \boldsymbol{d})^{1 / 2} \\
& =(1 / \boldsymbol{r}) \log A / \varepsilon, \quad A=(\varrho / \boldsymbol{\omega d})^{1 / 2} .
\end{aligned}
$$

A better estimation can be obtained, if the $O_{i}$ are assumed to be orthogonal. Then we get

$$
\begin{aligned}
\left\|e^{-\boldsymbol{M} t} W^{\prime}(0)\right\|^{2} & =\sum e^{-2 r_{i} t}\left|\gamma_{i}\right|^{2} \\
& \leqq e^{-2 \boldsymbol{r} t}\left\|W^{\prime}(0)\right\|^{2} \\
& \leqq e^{-2 \boldsymbol{r} t} / \boldsymbol{d} .
\end{aligned}
$$

In order to get measurable changes, we must demand

$$
1 / d-1 / d \geqq \varepsilon^{2} .
$$

\section{Proof of Equation (10) for $X \in \mathfrak{M}$}

$$
X \in \mathfrak{M} \Rightarrow X=\sum \gamma_{i} O_{i} .
$$

Now $\eta$ can be found in such a manner that

$$
W \text { eq }+\eta O_{i}=V
$$

fulfills all properties of a statistical operator. It follows from the properties (5) that

$$
\operatorname{Sp} O_{i}=\left(O_{i} ; P \mathfrak{g}\right)=0 .
$$

Hence $\operatorname{Sp} V=1$. We must demand that $V>0$. This can be fulfilled with a sufficiently small $\eta$. Therefore Eq. (10) is valid for $W^{\prime}(0)=\eta O_{i}$, hence for $O_{i}$ itself and therefore on $\mathfrak{M}$.

D. Properties of $\boldsymbol{M}$, Proof of: $\left\|W^{\prime}(0)\right\|^{2} \leqq 1 / \boldsymbol{d}-1 / d$ The formal solution of Eq. (4) is $\tilde{W}(t)=e^{-\boldsymbol{M} t} W(0)$. Two questions arise:

1. $\tilde{W}(t)=\tilde{W}^{+}(t)$ ?

2. $\tilde{W}(t)>0$ ?
We have

$$
\begin{aligned}
& \tilde{W}(t)=W^{\text {eq }}+\sum \gamma_{i} e^{-\lambda_{i} t} O_{i}, \\
& \tilde{W}^{+}(t)=W^{\text {eq }}+\sum \gamma_{i} e^{-\lambda_{i}{ }^{*} t} O_{i}{ }^{+} .
\end{aligned}
$$

Let be $\boldsymbol{S}$ the operator with eigenvalues $\lambda_{i}{ }^{*}$ and the eigenvectors $\mathrm{O}_{i}^{+}$:

$$
\begin{array}{ll}
\boldsymbol{S} O_{i}^{+}=\lambda_{i} * O_{i}+, & \boldsymbol{S} P_{\mathfrak{g}}=0, \\
\boldsymbol{M} O_{i}=\lambda_{i} O_{i}, & \boldsymbol{M} P_{\mathfrak{g}}=0 .
\end{array}
$$

It is easily seen that $\left(P_{r} ; \boldsymbol{S} P_{l}\right)=\left(P_{r} ; \boldsymbol{M} P_{l}\right)^{*}$. Now, if $\tilde{W}^{+}=\tilde{W}$, we have

$$
\begin{aligned}
& {\left[e^{-\boldsymbol{M} t}-e^{-\boldsymbol{S} t}\right] W(0)=0 \Rightarrow \boldsymbol{M}=\boldsymbol{S} \Rightarrow} \\
& \left(P_{r} ; \boldsymbol{M} P_{l}\right)=\left(P_{r} ; \boldsymbol{M} P_{l}\right)^{*} .
\end{aligned}
$$

Thus: A necessary and sufficient condition for $\tilde{W}=\tilde{W}^{+}$is, that the matrix elements $\left(P_{r} ; \boldsymbol{M} P_{l}\right)$ are real numbers. Now let us answer the second question. We can write

$$
\tilde{W}(t)=\sum \lambda_{i}(t) P_{i} .
$$

Hence

$$
\begin{aligned}
& \sum \dot{\lambda}_{i} P_{i}=-\boldsymbol{M} \sum \lambda_{j} P_{j} \Rightarrow \\
& \dot{\lambda}_{j}=-1 / d_{j} \sum M_{j i} \lambda_{i}, \quad M_{j i}=\left(P_{j} ; \boldsymbol{M} P_{i}\right) .
\end{aligned}
$$

Let be $t$ the first time with

$$
\lambda_{i_{1}}(t)=\lambda_{i_{2}}(t)=\cdots \lambda_{i_{k}}(t)=0,
$$

all other $\lambda_{j}(t)>0$.

Then we get

$$
\dot{\lambda}_{i_{r}}=-1 / d_{i_{r}} \sum M_{i_{r} r} \lambda_{r} .
$$

Therefore all $\lambda_{i}$ remain positive in time, if $M_{i j} \leqq 0$ for $i \neq j$, and this yields $\tilde{W}(t)>0$.

From $\boldsymbol{S}=\boldsymbol{M}$ we get: If $O$ is an eigenoperator of $\boldsymbol{M}$, then $O^{+}$too, if $\lambda$ is an eigenvalue of $\boldsymbol{M}$, then $\lambda^{*}$ too.

$$
\text { Proof of: }\left\|W^{\prime}(0)\right\|^{2} \leqq 1 / d-1 / d
$$

We have

and

$$
\begin{aligned}
\left\|W^{\prime}(0)\right\|^{2} & =\operatorname{Sp}\left(\left(\sum w_{i} P_{i}-P_{5} / d\right)^{2}\right) \\
& =\sum\left(w_{i}-1 / d\right)^{2} d_{i}
\end{aligned}
$$

$$
\sum w_{i} d_{i}=1, \quad 0 \leqq w_{i} \leqq 1 / d_{i} .
$$

The usual formalism yields an extremum $\boldsymbol{w}_{i}=1 / d$ or $W^{\prime}(0)=0$.

Hence the maximum must be on the boundary of the set

$$
B=\left\{\left(w_{1} \ldots w_{\varrho}\right) \mid \sum w_{i} d_{i}=1,0 \leqq w_{i} \leqq 1 / d_{i}\right\} .
$$


If, for instance, $w_{j}=1 / d_{j}$ all other $w_{i}$ vanish. Let us therefore consider the case that some of the $w_{i}=0$, while the remaining $w_{j}$ are free variables.

Then we get by differentiation

$$
\boldsymbol{w}_{j}=1 / d^{\prime}, \quad d^{\prime}=\sum^{\prime} d_{j},
$$

hence

$$
\left\|W^{\prime}(0)\right\|^{2}=\left(1 / d^{\prime}-1 / d\right)^{2} d^{\prime}+(1 / d)^{2}\left(d-d^{\prime}\right),
$$

or

$$
\left\|W^{\prime}(0)\right\|^{2} \leqq 1 / d-1 / d
$$

where $\boldsymbol{d}$ is the minimal dimension $d_{i}$.

\section{E. Proof of a Lemma}

Let be

$$
\left\|\left(\boldsymbol{M}-\int_{0}^{\tau^{*}} \boldsymbol{K}(\tau) \mathrm{d} \tau\right) W\right\| \leqq \varepsilon\|\boldsymbol{M} W\|
$$

for all $W$.

Then we get

$$
\begin{aligned}
& \left\|\left(\boldsymbol{M}-\int_{0}^{\tau^{*}} \boldsymbol{K}(\tau) \mathrm{d} \tau\right) X\right\| \\
& \quad=\|\boldsymbol{D} X\| \leqq \varepsilon\|\boldsymbol{M}\|\|X\|(\delta \bar{d})^{1 / 2}, \\
& \delta=\sum 1 / d_{i}, \quad \bar{d}=d_{\max } .
\end{aligned}
$$

Proof:

$$
\begin{aligned}
& X=\sum \sigma_{\alpha} W_{\alpha} \Rightarrow\|\boldsymbol{D} X\|=\left\|\sum \sigma_{\alpha} \boldsymbol{D} W_{\alpha}\right\| ; \\
& \left\|\sum \sigma_{\alpha} \boldsymbol{D} W_{\alpha}\right\| \leqq \varepsilon\|\boldsymbol{M}\| \sum \sigma_{\alpha}\left\|W_{\alpha}\right\| ;
\end{aligned}
$$

[1] S. Großmann, Funktionalanalysis, Akademische Verlagsanstalt, Frankfurt (Main) $\mathbf{1 9 7 0}$.

[2] O. Seeberg, Strong Causality, Localizability and Nonlinearity of the Transport Equations for Macroscopic Observables (to be published).

$$
\begin{aligned}
& \sum \sigma_{\alpha}\left\|W_{\alpha}\right\|=\sum\left|\sigma_{\alpha}\right| d_{\alpha}{ }^{-1 / 2} \Rightarrow \\
& \sum\left|\sigma_{\alpha}\right|\left\|W_{\alpha}\right\| \leqq\left[\sum_{\alpha}\left|\sigma_{\alpha}\right|^{2} \sum_{\beta} 1 / d_{\beta}\right]^{1 / 2} \\
& =\left[\sum_{\alpha}\left|\sigma_{\alpha}\right|^{2} \delta\right]^{1 / 2} ; \\
& \sum\left|\sigma_{\alpha}\right|^{2} \leqq \sum\left|\sigma_{\alpha}\right|^{2} / \mathrm{d} \alpha \bar{d} \Rightarrow \\
& \|D X\| \leqq \varepsilon\|\boldsymbol{M}\|\|X\|(\delta \bar{d})^{1 / 2}
\end{aligned}
$$

F. Estimation of $\int_{\tau^{*}}^{t}\left\|e^{-\boldsymbol{M}(t-\tau)} \boldsymbol{M} W^{\prime}(0)\right\| \mathrm{d} \tau$

We have

$$
\begin{aligned}
& \left\|e^{-\boldsymbol{M}(t-\tau)} \boldsymbol{M} W^{\prime}(0)\right\| \\
& \leqq\|\boldsymbol{M}\|\left\|e^{-\boldsymbol{M}(t-\tau)} W^{\prime}(0)\right\| \\
& \leqq\|\boldsymbol{M}\|(\varrho / \boldsymbol{\omega} \boldsymbol{d})^{1 / 2} e^{-\boldsymbol{r}(t-\tau)} \text {, }
\end{aligned}
$$

hence

$$
\int_{\tau^{*}}^{t}\left\|e^{-M(t-\tau)} M W^{\prime}(0)\right\| \mathrm{d} \tau \leqq\|\boldsymbol{M}\|(\varrho / \boldsymbol{\omega} d)^{1 / 2} 1 / \boldsymbol{r},
$$

or, with the better estimation in Appendix (B):

$$
\int_{\tau^{*}}^{t}\left\|e^{-\boldsymbol{M}(t-\tau)} M W^{\prime}(0)\right\| \mathrm{d} \tau \leqq\|\boldsymbol{M}\|(1 / \boldsymbol{d})^{1 / 2} 1 / \boldsymbol{r} .
$$

\section{Acknowledgements}

I wish to thank Professor Dr. R. Jelitto from the University of Frankfurt (Main) for helpful remarks during the course of a seminar held at Frankfurt.

[3] R. Zwanzig, Lect. in Theor. Physics 4, 106 (1960).

[4] G. Ludwig, Z. Phys. 173, 2, 232 (1963).

[5] R. Zwanzig, J. Chem. Phys. 40, 9 (1964).

[6] K. Kawasaki, Phys. Rev. 148, 1 (1966). 